\title{
Functional magnetic resonance imaging evaluation of central sensitization in an experimental model of pain in rats with induced endometriosis
}

\author{
Julio Cesar Rosa-e-Silva, Helmer Herren, Juliana Meola, Omero Benedicto Poli-Neto, Ana Carolina \\ Japur de Sá Rosa-e-Silva
}

Department of Gynecology and Obstetrics, School of Medicine of Ribeirão Preto, University of São Paulo, Brazil

Correspondence to: Julio Cesar Rosa e Silva. Associate Professor, Department of Gynecology and Obstetrics, School of Medicine of Ribeirão Preto, University of São Paulo, 14049-900 Ribeirão Preto, SP, Brazil. Email: juliocrs@usp.br.

Comment on: Zheng P, Mei J, Leng J, et al. Evaluation of the brain functional activities in rats various location-endometriosis pain model. Ann Transl Med 2019;7:767.

Submitted Jun 22, 2020. Accepted for publication Jul 20, 2020.

doi: 10.21037/atm-2020-118

View this article at: http://dx.doi.org/10.21037/atm-2020-118

Chronic pelvic pain (CPP) is a common clinical manifestation of endometriosis. Up to $87 \%$ of women with CPP presented endometriosis lesions at laparoscopy (1). It is not rare that CPP in patients with endometriosis is frequently refractory to clinical and surgical treatments. While the aim of these therapies is to control the inflammatory process that occur as a consequence of the presence of ectopic endometrial lesions, they do not directly target pain due to central sensitization of the nervous system and myofascial dysfunction, which can perpetuate pain from myofascial trigger points even if endometriosis lesions are destroyed or shrinked after treatment. The mechanisms responsible for CPP is development this population still to be fully described. As in most chronic pain syndromes, there is no correlation between the presence or extension of organic pathologies and the severity of symptoms, and many women with severe endometriosis experience little or no pain (2). Until now, the available therapies for endometriosis are not efficient as a definitive therapy, they can produce satisfactory results in terms of controlling the progression of the disease and reducing lesions activity (inflammatory process). However, it is not uncommon that pain occurs, even with no detectable residual pelvic pathology (3).

The hypothesis that central pain amplification might be one of the mechanisms of CPP has been addressed and supported in recent studies. The chronic stimuli of nociceptors lead to lower activation threshold and peripheral sensitization. The profusion of noxious stimuli from the damaged tissues into the dorsal horn of the spinal cord induces structural and functional changes in the central nervous system (CNS), ultimately leading to central sensitization and causing exaggerated responses to peripheral stimuli(4).

Sensorial alterations such as hyperalgesia and allodynia in and outside the painful areas have been described in patients with different chronic pain syndrome. Also increased neural activity and structural alterations in pain-related cortical areas have been pointed as evidence of CNS abnormalities in pain processing in these cases $(5,6)$.

Pain is intrinsically salient and, similarly to other sensorial perceptions, has a specific signature in the brain described as the dynamic pain connectome. The recognition of these specific signals and its characterization have been widely studied using the resting-state functional magnetic resonance imaging (rsfMRI), which enables functional interactions in the human brain connectome (7). It measures ongoing brain activity by enabling the recording of the Blood Oxygenation Level Dependent (BOLD) signals and the functional connectivity (FC). Studies applying this method associated to multivariate pattern analysis (MVPA) have recently documented brain activity supposed to be specific to pain, revealing network-level patterns of activity that can probably distinguish, with high sensitivity and specificity (often $>90 \%$ ), pain from nonpainful warmth, pain anticipation, pain recall, and the experience of social rejection $(8,9)$. 
Brain works as a network system with different units that have specific characteristics according to its remote neuronal connection and what function it commands. FC was initially defined as the interregional relationship between remote brain regions and employed Pearson's correlation coefficient between their BOLD time series (10). FC may be classified according to the spatial scale to measure functional interactions or synchronizations between the neighboring voxels or vertices. It is designated as local $\mathrm{FC}$ when these interactions occur between 10 and $15 \mathrm{~mm}$. Thus, local FC and remote FC can influence each other, showing the importance of investigating local FC with local functional homogeneity (11).

Due to the its composition (high phospholipid concentration) the mammalian neural tissues are easily damaged by free radicals, including reactive nitrogen species (RNS) and reactive oxygen species (ROS). Also, neuronal antioxidant defenses are weak (12). Thus, several clinical conditions lead to increased ROS/RNS levels and impair biomolecules, organelles and antioxidant defenses, promoting oxidative stress, mitochondrial dysfunction and inflammation (13). This negative impact of CPP in brain organic composition is supported by studies that demonstrated a reduction in gray matter volume (GMV) in pain regulatory regions such as the thalamus, cingulate gyrus, putamen, and insula, despite da presence of endometriosis. Specifically in endometriosis-associated CPP GMV is reduced in the thalamus, mid-cingulate cortex, and posterior insula, what have been associated to worse pain displeasure (6).

In Annals of Translational Medicine, Zheng et al. described the results of an experimental synergistic analysis of the higher-position central sensitization mechanism in a rat model of induced endometriosis (14). It proposed to analyze the impact of pain, secondary to various locations of endometriosis (EM), in the generation of images using CNS rsfMRI. For this purpose, the rats were submitted to three different types of induced endometriosis by suturing endometrial tissue to: the abdominal wall $(\mathrm{n}=5)$, the gastrocnemius $(n=5)$, and the ovary $(n=5)$; they also included a sham control group $(n=5)$. They measured the pain sensitization by hotplate test in a synergistic analysis with magnetic resonance scan evaluation of regional homogeneity ( $\mathrm{ReHo}$ ) at 4, 8, and 12 weeks after endometriosis induction, followed by histological analysis of the CNS by Nissl staining method. They found: a higher pain sensitization in abdominal wall EM and in gastrocnemius EM than in the ovary EM group and sham control; the right thalamus and the left olfactory tubercle ReHo values were lower in gastrocnemius EM compared to the other three groups; he gastrocnemius EM animals, in comparison to the other three groups, presented with a decreased number of neurons, cavitation around the nucleus and pyknotic homogenous nuclei in the right thalamus; they also found Nissl bodies with irregular shape; finally there was no difference between the 4 groups in relation to the left olfactory tubercle.

The authors conclude that the central sensitization mechanism of EM, in various location, is probably located in thalamus. They also suggest that gastrocnemius EM animals with more severe pain, the oxidative activation may be weakened in the thalamus. Based on their findings, the authors recommended that this data could support future research on imageology and pathology of various location of EM pain.

The study supports the idea that ReHo can be used as an index of network centrality, indicating its importance in human connectome, it can be characterized as the local FC between a particular node and its surrounding nodes. Pieces of evidence suggest that the "network" of brain regions involved in a pain response is far less pain-specific than the neuroimaging community had assumed. They suggest that there may be an abnormal activation of these networks by stimuli that might be pain-related but are not necessarily painful, and this may be a common pattern among chronic pain syndromes (14). In a study conducted by Shimo et al., 2011 (15), patients with low back pain (LBP) were exposed to pictures that supposedly triggered painful events; the patients reported increased back pain during the exposure to the images. Another study exposed patients with chronic migraine to pain-related words while they were submitted to brain scan and, although they were asymptomatic at the time of scan, they presented increased activations of the anterior insula and orbitofrontal cortex. As-Sanie et al. found that higher anterior insula connectivity to $\mathrm{mPFC}$ was correlated with anxiety scores [STPIDA-A, $r(12)=0.61$, $\mathrm{P}=0.02$ ], depression scores [STPIDA-D, $\mathrm{r}(12)=0.60, \mathrm{P}=0.03$ ] and clinical pain intensity [BPI, $\mathrm{r}(11)=0.55, \mathrm{P}=0.05]$. According to Schmidt-Wilcke, "pain neuromatrix" may be described as a salience network, and it is disrupted or dysregulated in chronic pain with an altered "baseline" (6).

Pain, has a specific 'neural signature' that can be described as the dynamic pain connectome. The identification of this neural representation can be extremely tricky once a pain-related but non-painful stimulus can activate the same brain area and evoke a similar fMRI brain 
image. In an opinion report, Kucyi and Davis describe pain as an intrinsically dynamic experience and the codification of 'pain connectome' as "the spatiotemporal signature of brain network communication that represents the integration of all cognitive, affective, and sensorimotor aspects of pain". The level of integration among all this aspects can vary spontaneously on multiple timescales (16). It has been demonstrated by imaging studies that distraction from a painful stimulus reduced the activation of the ascending pain system (17).

There are many questions if ReHo really reflects the local synchronization of spontaneous neural activity and, if by itself, it can represent the complete CNS response to pain stimuli. The study of Jiang and cols, 2014, examined regional differences in $\mathrm{ReHo}$ and stated that it truly represents a hierarchical organization of the brain and neurodevelopmental factors. However, a limitation of multivoxel pattern analysis (MVPA), using fMRI, is that it assesses brain activity as 'snapshots' in time, not considering pain connectome as a dynamic event, that must be analyzed and described beyond instantaneous patterns.

There is evidence that suggests that clinical pain perception is a result of an interaction between a dysfunctional pain regulatory system in the CNS and peripheral factors related to painful stimuli (18). The current literature suggests that even among visceral CPP conditions such as painful bladder syndrome and endometriosis, the perception of pain may be related to dysregulation or malfunction in motor cortical function and structure, even if it presents as hypersensitivity of the pelvic floor musculature and altered muscle activity. When dealing with women with endometriosis-associated chronic pain, one has to also take into account the environmental stressors, genetic factors and cognitive/behavioral factors that likely modulate chronic pain (6).

These findings show that the complexity of the networks involved in central sensitization leading to chronic pain are many and modulated by other information that shares the same channels and acts in a dynamic network of interactions. Although painful experimental stimuli are not exactly comparable to the experience of chronic pain, it allows the identification of the neural areas that are activated by these stimuli and bring insights into the central processes involved in pain perception, especially when comparing patients with chronic pain and healthy pain-free controls. In it important to highlight that proper control condition in longitudinal assessments are essential for an accurate determination of pain connectome dynamics and to identify the mechanism of action of different pain therapies (16). This study focuses only on using ReHo and anatomical alteration in the CNS detected through Nissel staining methods, giving a reductionist approach to the topic. However, it is of relevance in the creation of a study model of treatments for endometriosis-associated chronic pain.

\section{Acknowledgments}

Funding: None.

\section{Footnote}

Provenance and Peer Review: This article was commissioned by the editorial office, Annals of Translational Medicine. The article did not undergo external peer review.

Conflicts of Interest: All authors have completed the ICMJE uniform disclosure form (available at http://dx.doi. org/10.21037/atm-2020-118). The authors have no conflicts of interest to declare.

Ethical Statement: The authors are accountable for all aspects of the work in ensuring that questions related to the accuracy or integrity of any part of the work are appropriately investigated and resolved.

Open Access Statement: This is an Open Access article distributed in accordance with the Creative Commons Attribution-NonCommercial-NoDerivs 4.0 International License (CC BY-NC-ND 4.0), which permits the noncommercial replication and distribution of the article with the strict proviso that no changes or edits are made and the original work is properly cited (including links to both the formal publication through the relevant DOI and the license). See: https://creativecommons.org/licenses/by-nc-nd/4.0/.

\section{References}

1. Practice Bulletin No. 114: Management of Endometriosis. Obstet Gynecol 2010;116:223-36.

2. Fedele L, Parazzini F, Bianchi S, et al. Stage and Localization of Pelvic Endometriosis and Pain. Fertil Steril 1990;53:155-8.

3. Shakiba K, Bena JF, McGill KM, et al. Surgical Treatment of Endometriosis: A 7-year Follow-Up on the Requirement for Further Surgery. Obstet Gynecol 2008;111:1285-92. 
4. Aredo JV, Heyrana KJ, Karp BI, et al. Relating Chronic Pelvic Pain and Endometriosis to Signs of Sensitization and Myofascial Pain and Dysfunction. Semin Reprod Med 2017;35:88-97.

5. Schmidt-Wilcke T. Variations in Brain Volume and Regional Morphology Associated With Chronic Pain. Curr Rheumatol Rep 2008;10:467-74.

6. As-Sanie S, Kim J, Schmidt-Wilcke T, et al. Functional connectivity is associated with altered brain chemistry in women with endometriosis-associated chronic pelvic pain HHS Public Access. J Pain 2016;17:1-13.

7. Zuo XN, Xing XX. Test-retest Reliabilities of RestingState FMRI Measurements in Human Brain Functional Connectomics: A Systems Neuroscience Perspective. Neurosci Biobehav Rev 2014;45:100-18.

8. Wager TD, Atlas LY, Lindquist MA, et al. An fMRI-Based Neurologic Signature of Physical Pain. N Engl J Med 2013;368:1388-97.

9. Liang M, Mouraux A, Hu L, et al. Primary sensory cortices contain distinguishable spatial patterns of activity for each sense. Nat Commun 2013;4:1979.

10. Friston KJ, Frith CD, Liddle PF, et al. Functional Connectivity: The Principal-Component Analysis of Large (PET) Data Sets. J Cereb Blood Flow Metab 1993;13:5-14.
11. Jiang L, Zuo XN. Regional Homogeneity: A Multimodal, Multiscale Neuroimaging Marker of the Human Connectome. Neuroscientist 2016;22:486-505.

12. Areti A, Yerra VG, Naidu V, et al. Oxidative stress and nerve damage: Role in chemotherapy induced peripheral neuropathy. Redox Biol 2014;2:289-95.

13. Carrasco C, Naziroğlu M, Rodríguez AB, et al. Neuropathic Pain: Delving into the Oxidative Origin and the Possible Implication of Transient Receptor Potential Channels. Front Physiol 2018;9:95.

14. Borsook D, Edwards R, Elman I, et al. Pain and Analgesia: The Value of Salience Circuits. Prog Neurobiol 2013;104:93-105.

15. Shimo K, Ueno T, Younger J, et al. Visualization of painful experiences believed to trigger the activation of affective and emotional brain regions in subjects with low back pain. PLoS One. 2011;6:e26681.

16. Kucyi A, Davis KD. The Dynamic Pain Connectome. Trends Neurosci 2015;38:86-95.

17. Bushnell MC, Ceko M, Low LA. Cognitive and emotional control of pain and its disruption in chronic pain. Nat Rev Neurosci 2013;14:502-11.

18. Tu FF, As-Sanie S. A modest proposal to investigate chronic uterine pain. BJOG 2017;124:182-4.
Cite this article as: Rosa-e-Silva JC, Herren H, Meola J, Poli-Neto OB, Rosa-e-Silva ACJS. Functional magnetic resonance imaging evaluation of central sensitization in an experimental model of pain in rats with induced endometriosis. Ann Transl Med 2020;8(20):1286. doi: 10.21037/atm-2020-118 